\title{
Pd-tetraethyl tetra-t-butylcalixarene-tetra(oxyacetate) complex catalyzed acylodeboronation of arylboronic acids with benzoyl chloride or acetic anhydride
}

\author{
Jinli Zhang, Zixing Han, Junmiao Li, and Yangjie Wu* \\ The College of Chemistry and Molecular Engineering, Henan Key Laboratory of Chemical \\ Biology and Organic Chemistry, Key Laboratory of Applied Chemistry of Henan Universities, \\ Zhengzhou University, Zhengzhou, 450052, P. R. China \\ E-mail:wyj@zzu.edu.cn
}

\begin{abstract}
The palladium-tetraethyl tetra-t-butylcalixarene-tetra(oxyacetate) complex, in the form of $[\mathrm{NaL}]^{+}$ $\left[\mathrm{PdCl}_{3}\right]^{-}$, exhibited high catalytic efficiency for the acylodeboronation reaction of arylboronic acids with benzoyl chloride or acetic anhydride, producing the corresponding ketones in good to excellent yields under mild reaction conditions. Various arylboronic acids, benzoyl chlorides and acetic anhydrides were tolerated in this method. Moreover, a typical method for synthesis of acetyl aryl ketones was obtained.
\end{abstract}

Keywords: Tetraethyl tetra-t-butylcalixarene-tetra(oxyacetate), palladium catalyst, aryl ketone, carbonylation

\section{Introduction}

Due to the wide occurrence of aryl ketone moiety in a large number of biologically active compounds ${ }^{1}$, natural products, ${ }^{2}$ cosmetics, ${ }^{3}$ pharmaceutical, fragrance, dye, agrochemicals and functional material industries as well as in organic synthesis, ${ }^{4}$ considerable efforts have been made for its synthesis. Aromatic ketones can be effectively prepared by palladium catalyzed carbonylation reaction of acyl chlorides with boronic acid. ${ }^{5-7}$ Such method can avoid the restrictions of the regioselectivity limitation of para position (traditional Friedel-Crafts acylation $^{8}$ ), using of toxic carbon monoxide and the formation of biaryl side products (carbonylative Suzuki-Miyaura cross-coupling ${ }^{9}$ ), the requirement of directing group (carbonylative $\mathrm{C}-\mathrm{H}$ activation ${ }^{10}$ ), harsh reaction conditions (oxidation of benzyl alcohols ${ }^{11}$ and addition of organometallic reagents to carboxylic derivatives or nitriles ${ }^{12}$ ) and incompatibility with electron-deficient groups etc. ${ }^{7,13-15}$ 
However, there are still some limitations for the synthesis of aliphatic acyl phenone using aliphatic acyl chloride or acetic anhydride as acyl reagent, especially for the synthesis of various acetophenone from acetic chloride or anhydride. ${ }^{5,5 f, 6 \mathrm{~g}, 7 \mathrm{a}}$ The coupling between phenylboronic acid and acetic anhydride were scatteredly reported ${ }^{5 \mathrm{c}, 5 \mathrm{f}, 6 \mathrm{~d}}$ and only Gooben ${ }^{5 \mathrm{f}}$ obtained satisfactory results. Thus, it is challenging to develop catalytic carbonylation of aliphatic acyl chloride or acetic anhydride with high efficiency.

Nowadays, calixarenes are emerging as a versatile family of ancillary ligands in a variety of catalytic processes. ${ }^{16}$ For example, calixarenes bearing phosphines or NHCs functional groups have been reported to exhibit notably high catalytic activities in transition metal catalyzed C-C bond formation reactions. ${ }^{17}$ In addition, calix[4]aryl acetates showed remarkably high sodium ion affinity and selectivity owing to the "size fitting effect". ${ }^{18 a-18 c}$ Recently, our group reported the synthesis of a kind of tetraethyl tetra-t-butylcalixarene-tetra(oxyacetate) and its catalytic activity in Suzuki cross-coupling reaction. ${ }^{18 \mathrm{~d}}$ In this paper, we wish to report the palladiumtetraethyl tetra- $t$-butylcalixarene-tetra(oxyacetate) complex catalyzed acylodeboronation reaction of arylboronic acids with benzoyl chloride or acetic anhydride respectively for the easy preparation of aryl ketones. To the best of our knowledge, this is the first report on the coupling of carboxylic anhydride or acyl chloride catalyzed by $0.5 \%$ catalyst loading of palladium complex based on tetraethyl tetra-t-butylcalixarene-tetra(oxyacetate).

\section{Results and Discussion}

\section{Synthesis of the catalyst and its characterization}

Tetraethyl tetra-t-butylcalixarene-tetra(oxyacetate) (L) was synthesized according to the general procedure. ${ }^{19}$ Palladium complex was obtained according to the literature and characterized by Xray $^{18 \mathrm{~d}}$ analysis.

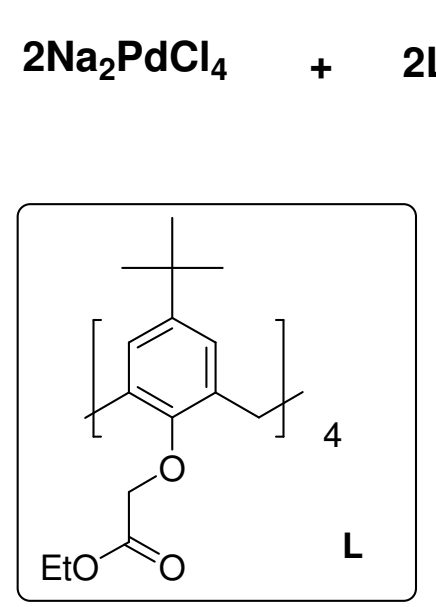

Scheme 1. Synthesis of catalyst 1.

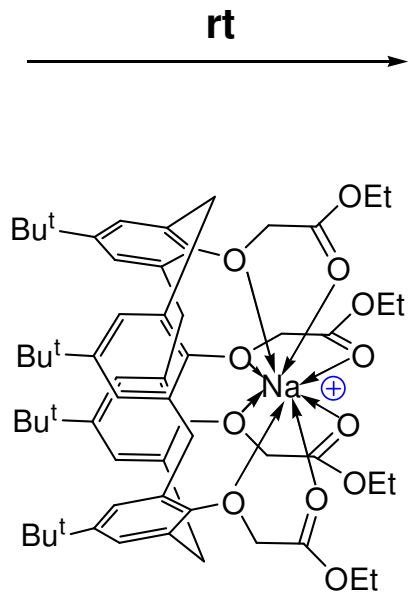

Cat. 1

\section{$2 \mathrm{NaCl}+2 \mathrm{NaLPdCl}_{3}$}

1

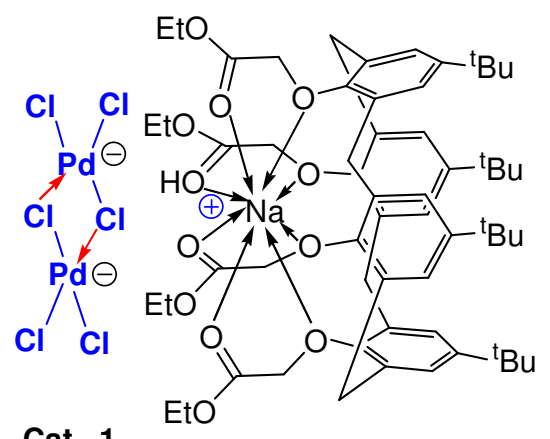


The red solid of $\mathbf{L}$ was obtained in a $65 \%$ yield. $\mathrm{mp}>280{ }^{\circ} \mathrm{C}(\mathrm{dec})$. NMR spectra: ${ }^{1} \mathrm{H}$ NMR $\left(\mathrm{CDCl}_{3}, 400 \mathrm{MHz}\right): \delta 7.07(8 \mathrm{H}, \mathrm{s}), 4.48-4.43(16 \mathrm{H}, \mathrm{m}), 4.26(4 \mathrm{H}, \mathrm{d}, J 12.2 \mathrm{~Hz}), 3.37(4 \mathrm{H}, \mathrm{d}, J$ $12.2 \mathrm{~Hz}), 1.47(12 \mathrm{H}, \mathrm{t}, J 7.1 \mathrm{~Hz}), 1.11(36 \mathrm{H}, \mathrm{s}) .{ }^{13} \mathrm{C}$ NMR: $\delta$ 171.0, 149.6, 148.2, 134.3, 125.7, $73.2,62.4,34.0,31.2,29.8,14.4$. As shown in Scheme 1, the $\mathrm{Na}_{2} \mathrm{Pd}_{2} \mathrm{Cl}_{6}$ complex with tetraethyl tetra-t-butyl-calixarene-tetra(oxyacetate) shows clearly a "cone" structure.

\section{Catalytic survey}

\section{Studies on acylodeboronation reaction of arylboronic acid with benzoyl chloride}

The coupling reaction of phenyl boronic acid and benzoyl chloride was chosen as a model reaction (Table 1) to test the reactivity of Cat. $\mathbf{1}$. To compare the catalytic activities, $\mathrm{Pd}(\mathrm{OAc})_{2}$ and $\mathrm{PdCl}_{2}$ were used under the same reaction conditions, and relatively lower yields were obtained respectively (entries 2-5). Yamamoto's and Goossen's studies have showed that the phosphine ligands played key roles in the successful execution of the reactions. ${ }^{20}$ Gratifyingly, the carbonylation coupling proceeded smoothly and gave benzophenone in $68 \%$ isolated yield when Cat. 1 was used as a catalyst in the presence of $\mathrm{PPh}_{3}$ and $\mathrm{K}_{3} \mathrm{PO}_{4}$ in toluene at $90{ }^{\circ} \mathrm{C}$ under air (Table 1, entry 7). These results indicated that the combination of tetraethyl tetra-tbutylcalixarene-tetra(oxyacetate) and $\mathrm{PPh}_{3}$ promoted the procedure of acylodeboronation. Inspired by these results, we then screened the bases and reaction temperatures by using 0.5 mol\% Cat. 1. The carbonylation yield as high as $95 \%$ was obtained when the reaction was conducted at $70{ }^{\circ} \mathrm{C}$ under air (entry 10), which was much higher than the yields obtained by $\mathrm{Pd}\left(\mathrm{PPh}_{3}\right)_{4} \cdot{ }^{5 \mathrm{c}, 7 \mathrm{a}}$ Under the proper reaction conditions, other phosphine ligands were also applied and gave lower carbonylation yields (entries 11-14). Cat. 1 showed slight catalytic activities under lower temperature and concentration (entries 15-16). The results showed that the "size fitting effect" between tetraethyl tetra-t-butylcalixarene-tetra(oxyacetate) and palladium anion played key roles for the reaction (entry 18).

Table 1. Study on acylodeboronation reaction of phenyl boronic acid with benzoyl chloride

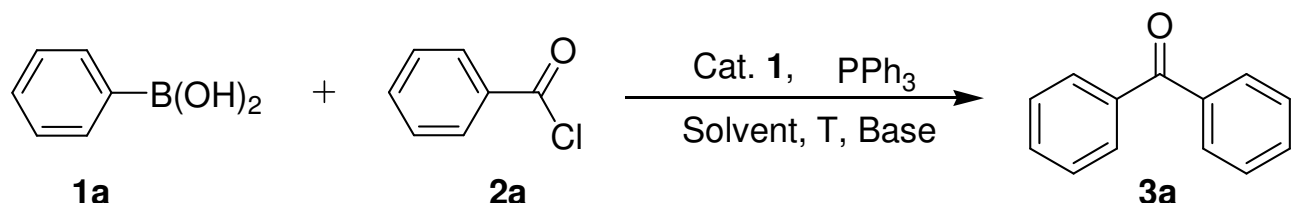

\begin{tabular}{cccccc}
\hline Entry $^{\mathrm{a}}$ & Catalyst & Bases & $\mathrm{T},{ }^{\circ} \mathrm{C}$ & Time, $\mathrm{h}$ & Yield \% \\
\hline 1 & -- & $\mathrm{K}_{3} \mathrm{PO}_{4}$ & 90 & 12 & Trace \\
2 & $\mathrm{Na}_{2} \mathrm{PdCl}_{4}+\mathrm{PPh}_{3}$ & $\mathrm{~K}_{3} \mathrm{PO}_{4}$ & 90 & 12 & $<5$ \\
3 & $\mathrm{Pd}(\mathrm{OAc})_{2}$ & $\mathrm{~K}_{3} \mathrm{PO}_{4}$ & 90 & 12 & 50 \\
4 & $\mathrm{Pd}(\mathrm{OAc})_{2}+\mathrm{PPh}_{3}$ & $\mathrm{~K}_{3} \mathrm{PO}_{4}$ & 90 & 12 & 31 \\
5 & $\mathrm{PdCl}_{2}$ & $\mathrm{~K}_{3} \mathrm{PO}_{4}$ & 90 & 12 & 16 \\
6 & $\mathrm{PdCl}_{2}+\mathrm{PPh}_{3}$ & $\mathrm{~K}_{3} \mathrm{PO}_{4}$ & 90 & 12 & 35 \\
\hline
\end{tabular}


Table 1. Continued

\begin{tabular}{|c|c|c|c|c|c|}
\hline Entry $^{\mathrm{a}}$ & Catalyst & Bases & $\mathrm{T},{ }^{\circ} \mathrm{C}$ & Time, $\mathrm{h}$ & Yield $\%^{\mathrm{b}}$ \\
\hline 7 & Cat. $1+\mathrm{PPh}_{3}$ & $\mathrm{~K}_{3} \mathrm{PO}_{4}$ & 90 & 12 & 68 \\
\hline 8 & Cat. $1+\mathrm{PPh}_{3}$ & $\mathrm{~K}_{2} \mathrm{CO}_{3}$ & 90 & 12 & 98 \\
\hline 9 & Cat. $1+\mathrm{PPh}_{3}$ & $\mathrm{Na}_{2} \mathrm{CO}_{3}$ & 90 & 12 & Trace \\
\hline 10 & Cat. $1+\mathbf{P P h}_{3}$ & $\mathrm{~K}_{2} \mathrm{CO}_{3}$ & 70 & 12 & 95 \\
\hline 11 & $\mathrm{Pd}(\mathrm{OAc})_{2}+\mathrm{dppe}$ & $\mathrm{K}_{2} \mathrm{CO}_{3}$ & 70 & 12 & 56 \\
\hline 12 & $\mathrm{Pd}(\mathrm{OAc})_{2}+\mathrm{dppf}$ & $\mathrm{K}_{2} \mathrm{CO}_{3}$ & 70 & 12 & 48 \\
\hline 13 & $\mathrm{PdCl}_{2}+$ dppe & $\mathrm{K}_{2} \mathrm{CO}_{3}$ & 70 & 12 & 77 \\
\hline 14 & $\mathrm{PdCl}_{2}+\mathrm{dppf}$ & $\mathrm{K}_{2} \mathrm{CO}_{3}$ & 70 & 12 & 71 \\
\hline 15 & Cat. $1+\mathrm{PPh}_{3}$ & $\mathrm{~K}_{2} \mathrm{CO}_{3}$ & 40 & 12 & 38 \\
\hline 16 & Cat. $1+\mathrm{PPh}_{3}$ & $\mathrm{~K}_{2} \mathrm{CO}_{3}$ & 70 & 12 & $76^{\mathrm{c}}$ \\
\hline 17 & Cat. $1+\mathrm{PPh}_{3}$ & $\mathrm{~K}_{2} \mathrm{CO}_{3}$ & 70 & 12 & $92^{\mathrm{d}}$ \\
\hline $18^{\mathrm{e}}$ & $\mathrm{L}+\mathrm{NaCl}+\mathrm{PdCl}_{2}$ & $\mathrm{~K}_{2} \mathrm{CO}_{3}$ & 70 & 12 & - \\
\hline
\end{tabular}

${ }^{a}$ benzoyl chloride $(1.0 \mathrm{mmol})$, phenyl boronic acid $(0.5 \mathrm{mmol})$, base $(1.0 \mathrm{mmol})$, complex $1(0.5$ mol\%, $3.1 \mathrm{mg}), \mathrm{PPh}_{3}(0.01 \mathrm{mmol})$ and toluene $(2.0 \mathrm{~mL}), 70{ }^{\circ} \mathrm{C} .{ }^{\mathrm{b}}$ isolated yields. ${ }^{\mathrm{c}} 0.25 \mathrm{~mol} \%$ complex 1 was used and the acylodeboronation yield was determined by GC analysis using 3a as internal standard substances. ${ }^{\mathrm{d}} 1 \mathrm{~mol} \%$ complex 1 was used and the acylodeboronation yield was determined by GC analysis using $\mathbf{3 a}$ as internal standard substances. ${ }^{\mathrm{e}}$ tetraethyl tetra-tbutylcalixarene-tetra(oxyacetate) $\mathbf{L}(0.005 \mathrm{mmol}), \mathrm{NaCl}(0.005 \mathrm{mmol}), \mathrm{PdCl}_{2}(0.005 \mathrm{mmol})$, and the acylodeboronation yield could not be repeated (20-60\%).

With the standard conditions in hand, we then examined the scope and limitation of Cat. 1 in the acylodeboronation reaction. Various arylboronic acids and benzoyl chloride were applied to this system (Table 2, entries 1-14). The results listed in Table 2 showed that this catalytic system tolerated various functional groups, such as $\mathrm{CH}_{3}, \mathrm{CH}_{3} \mathrm{O}, \mathrm{Br}, \mathrm{Cl}, \mathrm{F}, \mathrm{CHO}$, and $\mathrm{CH}_{3} \mathrm{CO}$. Both electron-rich and electron-deficient arylboronic acids could provide the desired products in moderate to excellent yields (Table 2, entries 1-10). 1-Naphthyl and 2-thienyl boronic acids also reacted smoothly with benzoyl chloride, yielding the corresponding aryl ketones in $89 \%$ and 95\% yields, respectively (Table 2, entries 11-12). Some ketones, such as $\mathbf{3 b}, \mathbf{3 f}, \mathbf{3 i}$ and $\mathbf{3 j}$, usually difficult to obtain from traditional Friedel-Crafts methods by using benzoyl chloride as substrate, ${ }^{8}$ were produced successfully with moderate to good yields in this protocol (Table 2, entries $2,6,9,10)$. 
Table 2. Complex 1 catalyzed acylodeboronation reaction of arylboronic acids with benzoyl chloride

$$
\text { 2a-c } \begin{gathered}
\text { Cat. } 1(0.5 \mathrm{~mol} \%) \\
\begin{array}{c}
\mathrm{K}_{2} \mathrm{CO}_{3}(2 \mathrm{eq}), 70^{\circ} \mathrm{C} \\
\text { Toluene, air }
\end{array}
\end{gathered}
$$

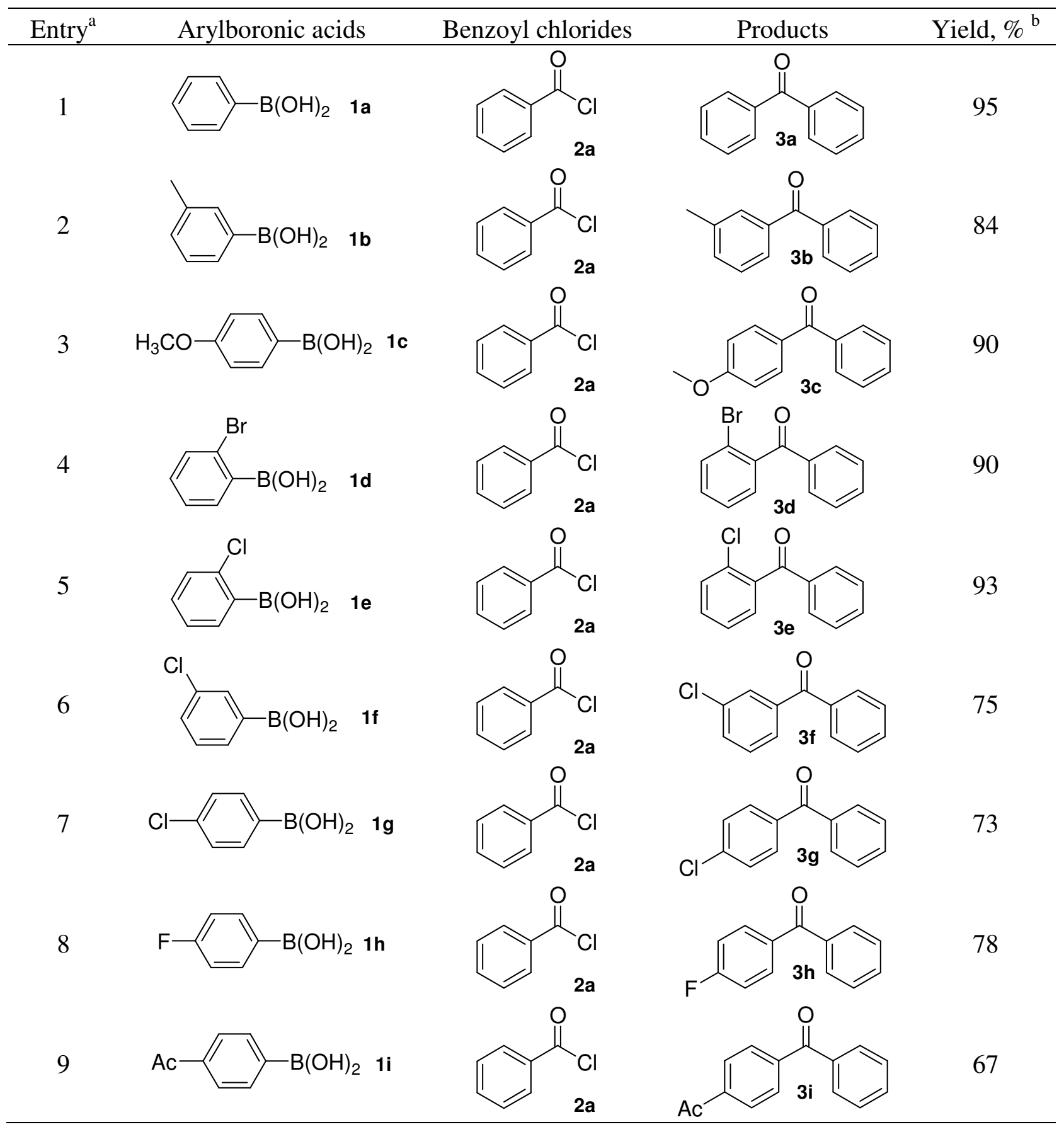


Table 2. Continued

Entrya

a reaction conditions: benzoyl chloride $(1.0 \mathrm{mmol})$, arylboronic acid $(0.5 \mathrm{mmol})$, base $(1.0$ mmol), complex $1(0.5 \mathrm{~mol} \%), \mathrm{PPh}_{3}(0.01 \mathrm{mmol})$ and toluene $(2.0 \mathrm{~mL}), 70{ }^{\circ} \mathrm{C}, 12 \mathrm{~h} .{ }^{\mathrm{b}}$ isolated yields.

\section{Studies on the acylodeboronation reaction of arylboronic acid with acetic anhydride}

When $\mathrm{Na}_{2} \mathrm{CO}_{3}$ was used as base (Table 1, entry 9), trace amount of benzophenone was observed. Benzoic acid anhydride was obtained as main product (determined by GC analysis). Treating the obtained benzoic acid anhydride with phenylboronic acid (new added), the desired benzophenone was isolated in $96 \%$ yield. These results revealed that the formation of ketone may proceed via the intermediate of benzoic acid anhydride, and Cat. 1 was also an efficient catalyst for the carbonylation reaction of arylboronic acid with acid anhydride (Scheme 2).

Acetophenone derivatives are useful intermediates in industry as well as in organic synthesis. Carbonylation reaction of acetyl chloride or anhydride with boronic acid could also be used for its synthesis. Bandgar ${ }^{6 \mathrm{~g}}$ has reported the reaction of arylboronic acid with benzoyl chloride by using $\mathrm{PdCl}_{2}$ as catalyst and $\mathrm{Na}_{2} \mathrm{CO}_{3}$ as base. But the catalyst loading is high (3.3\%) and the coupling between acetyl chloride with boronic acid remains unsuccessful ${ }^{6 \mathrm{~g}, 7 \mathrm{a}}$ or only in moderate yields. ${ }^{5 c}$ Also the coupling between acetic anhydride with arylboronic acid has been reported by Gooben ${ }^{5 f}$ by using $\mathrm{Pd}(\mathrm{OAc})_{2}$ as catalyst, expensive $\mathrm{P}(p-\mathrm{MeOPh})_{3}$ as ligand, and excess of arylboronic acid. 


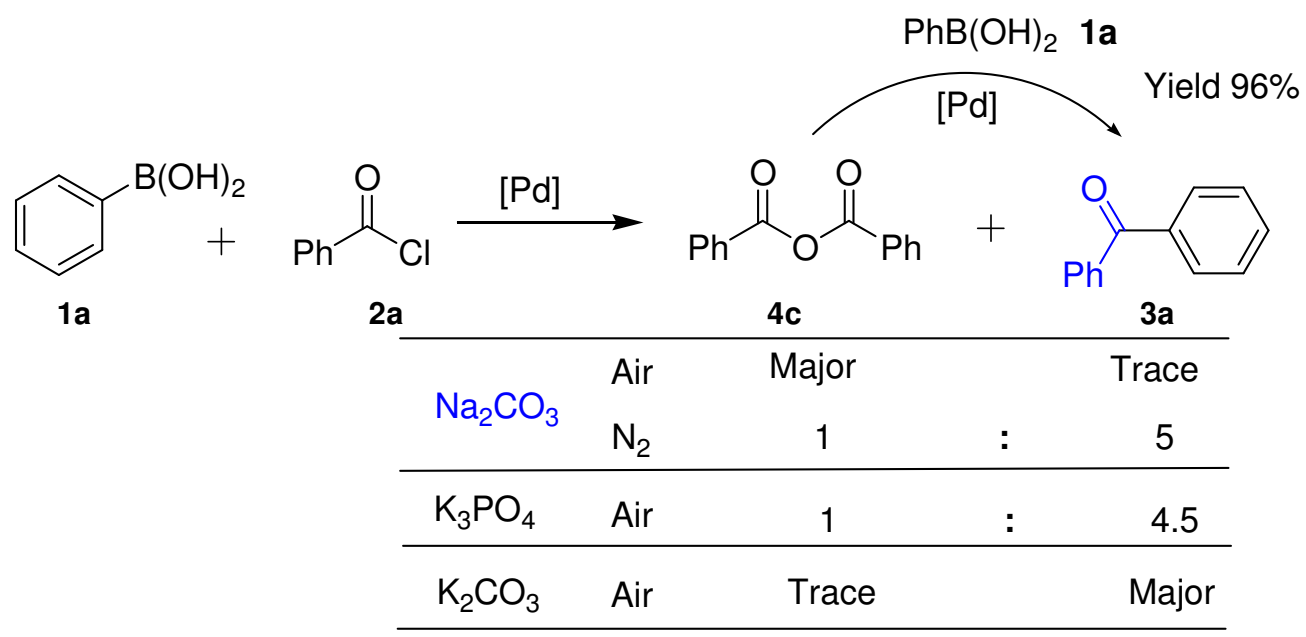

Scheme 2. Study on the effect of $\mathrm{Na}_{2} \mathrm{CO}_{3}$ as base for acylodeboronation reaction of phenylboronic acid with benzoic acid chloride.

We then choose acetic anhydride as reagent to test the reactivity of Cat.1. Initially, the crosscoupling reaction conditions of phenylboronic acid with acetic anhydride were screened (listed in Table 3). The best yield of $85 \%$ was obtained when $\mathrm{KHCO}_{3}$ was used as base in toluene at 110 ${ }^{\circ} \mathrm{C}$ under nitrogen (Table 3 , entry 6).

Tabel 3. Study on acylodeboronation reaction of phenyl boronic acid with acetic anhydride

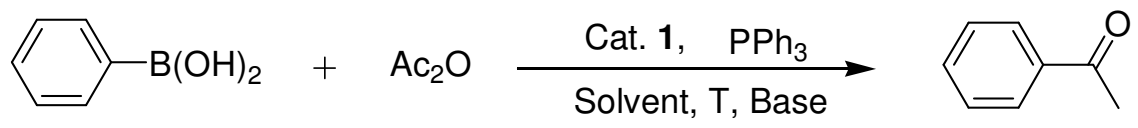

$1 \mathrm{a}$

$4 a$

$5 a$

\begin{tabular}{|c|c|c|c|c|c|c|c|}
\hline Entry $^{\mathrm{a}}$ & Catalyst & Solvent & Base & $\mathrm{T},{ }^{\circ} \mathrm{C}$ & Time, $\mathrm{h}$ & Gas & Yield, $\%^{\mathrm{b}}$ \\
\hline 1 & Cat. $1+\mathrm{PPh}_{3}$ & Toluene & $\mathrm{K}_{2} \mathrm{CO}_{3}$ & 70 & 12 & Air & 15 \\
\hline 2 & Cat. $1+\mathrm{PPh}_{3}$ & Toluene & $\mathrm{K}_{2} \mathrm{CO}_{3}$ & 70 & 12 & $\mathrm{~N}_{2}$ & 48 \\
\hline 3 & Cat. $1+\mathrm{PPh}_{3}$ & Toluene & $\mathrm{K}_{2} \mathrm{CO}_{3}$ & 110 & 3 & $\mathrm{~N}_{2}$ & 67 \\
\hline 4 & Cat. $1+\mathrm{PPh}_{3}$ & Toluene & $\mathrm{K}_{3} \mathrm{PO}_{4}$ & 110 & 3 & $\mathrm{~N}_{2}$ & 42 \\
\hline 5 & Cat. $1+\mathrm{PPh}_{3}$ & Toluene & $\mathrm{Na}_{2} \mathrm{CO}_{3}$ & 110 & 3 & $\mathrm{~N}_{2}$ & 40 \\
\hline 6 & Cat. $1+\mathrm{PPh}_{3}$ & Toluene & $\mathrm{KHCO}_{3}$ & 110 & 3 & $\mathbf{N}_{2}$ & 85 \\
\hline 7 & Cat. $1+\mathrm{PPh}_{3}$ & Toluene & $\mathrm{NaHCO}_{3}$ & 110 & 3 & $\mathrm{~N}_{2}$ & 17 \\
\hline 8 & $\mathrm{PdCl}_{2}+\mathrm{PPh}_{3}$ & Toluene & $\mathrm{Na}_{2} \mathrm{CO}_{3}$ & 110 & 3 & $\mathrm{~N}_{2}$ & 52 \\
\hline 9 & $\mathrm{PdCl}_{2}+\mathrm{dppe}$ & Toluene & $\mathrm{KHCO}_{3}$ & 110 & 3 & $\mathrm{~N}_{2}$ & Trace \\
\hline 10 & $\mathrm{PdCl}_{2}+\mathrm{dppf}$ & Toluene & $\mathrm{KHCO}_{3}$ & 110 & 3 & $\mathrm{~N}_{2}$ & Trace \\
\hline 11 & $\mathrm{Pd}(\mathrm{OAc})_{2}+\mathrm{PPh}_{3}$ & Toluene & $\mathrm{KHCO}_{3}$ & 110 & 3 & $\mathrm{~N}_{2}$ & 40 \\
\hline 12 & $\mathrm{Pd}(\mathrm{OAc})_{2}+\mathrm{dppe}$ & Toluene & $\mathrm{KHCO}_{3}$ & 110 & 3 & $\mathrm{~N}_{2}$ & Trace \\
\hline 13 & $\mathrm{Pd}(\mathrm{OAc})_{2}+\mathrm{dppf}$ & Toluene & $\mathrm{KHCO}_{3}$ & 110 & 3 & $\mathrm{~N}_{2}$ & Trace \\
\hline 14 & Cat. 1 & Toluene & $\mathrm{KHCO}_{3}$ & 110 & 3 & $\mathrm{~N}_{2}$ & Trace \\
\hline
\end{tabular}


Table 3. Continued

\begin{tabular}{cccccccc}
\hline Entry $^{\mathrm{a}}$ & Catalyst & Solvent & Base & $\mathrm{T},{ }^{\circ} \mathrm{C}$ & Time, h & Gas & Yield, \% ${ }^{\mathrm{b}}$ \\
\hline 15 & Cat. 1+PPh & THF & $\mathrm{KHCO}_{3}$ & 60 & 3 & $\mathrm{~N}_{2}$ & 77 \\
16 & Cat. 1+PPh & DMF & $\mathrm{KHCO}_{3}$ & 110 & 3 & $\mathrm{~N}_{2}$ & Trace \\
$17^{\mathrm{c}}$ & $\mathrm{L}+\mathrm{NaCl}+\mathrm{PdCl}_{2}$ & Toluene & $\mathrm{NaHCO}_{3}$ & 110 & 3 & $\mathrm{~N}_{2}$ & - \\
\hline
\end{tabular}

${ }^{a}$ acetic anhydride $(1.0 \mathrm{mmol})$, arylboronic acid $(0.5 \mathrm{mmol})$, base $(1.0 \mathrm{mmol})$, complex 1 (0.5 $\mathrm{mol} \%), \mathrm{PPh}_{3}(0.01 \mathrm{mmol})$ and solvent $(2.0 \mathrm{~mL}) .{ }^{\mathrm{b}}$ isolated yields. ${ }^{\mathrm{c}}$ yields could not be repeated $(20-40 \%)$.

Under the optimal conditions, the scope of arylboronic acids and anhydrides for this acylodeboronation transformation was investigated. As shown in Table 4, the carbonylation yields were influenced by electron effect. Arylboronic acids with electron-rich groups such as $\mathrm{CH}_{3}$ or $\mathrm{CH}_{3} \mathrm{O}$, as well as 1-naphthyl motif could be coupled with acetic anhydride in moderate to good yields (Table 4, entries 1-5). When arylboronic acids bearing electron-deficient groups were applied, moderate yields were still obtained (Table 4, entries 6-8). Other kinds of anhydrides were also applied, yielding the corresponding ketones in lower yields (Table 4, entries 9-10).

Table 4. Complex 1 catalyzed acylodeboronation reaction of arylboronic acids with acetic anhydride

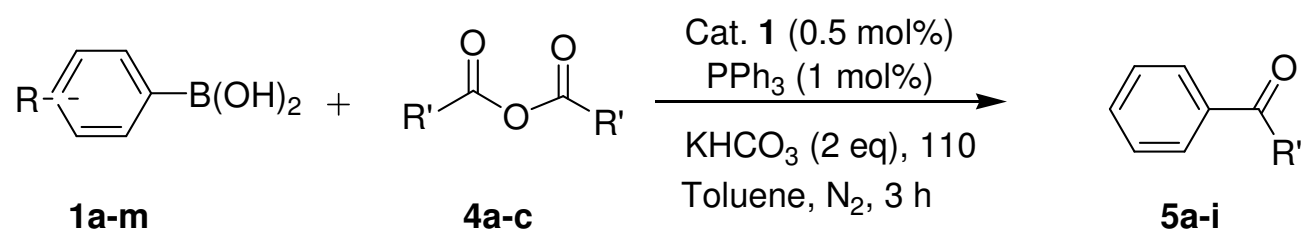

Entry $^{\mathrm{a}}$ Arylboronic acids


Table 4. Continued

Entry $^{\mathrm{a}}$

a reaction conditions: acetic anhydride $(1.0 \mathrm{mmol})$, arylboronic acid $(0.5 \mathrm{mmol})$, base (1.0 mmol), complex $1(0.5 \mathrm{~mol} \%), \mathrm{PPh}_{3}(0.01 \mathrm{mmol})$ and toluene $(2.0 \mathrm{~mL}), 110{ }^{\circ} \mathrm{C}, 3 \mathrm{~h} .{ }^{\mathrm{b}}$ isolated yields. ${ }^{\mathrm{c}} \mathrm{Na}_{2} \mathrm{CO}_{3}$ was used as base.

\section{Mechanistic study}

In order to provide evidence that the enhanced yields using Cat. 1 were not due to the use of a $\mathrm{Na}_{2} \mathrm{PdCl}_{4}$ instead of $\mathrm{Pd}(\mathrm{OAc})_{2}$ or $\mathrm{PdCl}_{2}$, the carbonylation reaction of benzoyl chloride with phenylboronic acid was conducted by using the mixture of $\mathrm{Na}_{2} \mathrm{PdCl}_{4}$ pre-catalyst and triphenylphosphine. It is clear that the high catalytic efficiency were attributed to the "size fitting effect" between tetraethyl tetra- $t$-butylcalixarene-tetra(oxyacetate) $\mathbf{L}$ and palladium anion (Table 5, entries 1-4). Furthermore, a investigation on the catalytic activity of $\mathrm{Na}_{2} \mathrm{PdCl}_{4}$ with different phosphine ligands such as $\mathrm{PPh}_{3}$, dppe or dppf was undertaken to clarify the role of tetraethyl $p$ tert-butyl-calix[4]aryl tetraacetate $(\mathbf{L})$ in the formation of the catalytically active species (Table 5, entries 5-7). $\mathrm{Na}_{2} \mathrm{PdCl}_{4}$ with the mixture of $\mathbf{L}$ and different phosphine as ligands showed slightly lower activity under the same reaction conditions. Except the ligand dppe, the mixture of $\mathrm{Na}_{2} \mathrm{PdCl}_{4}, \mathbf{L}$ and $\mathrm{PPh}_{3}$ or dppf resulted in the homo-coupling of phenylboronic acid. 
Table 5. Studies on the role of tetraethyl tetra-t-butylcalixarenetetra(oxyacetate) $\mathbf{L}$

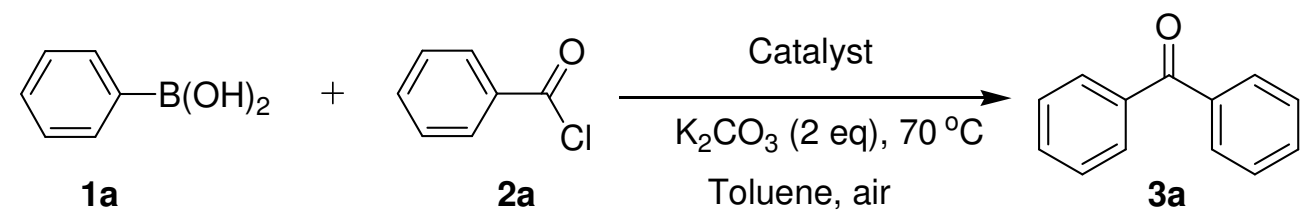

\begin{tabular}{ccc}
\hline Entry $^{\mathrm{a}}$ & Catalyst & ${\text { Yiled \% of } \mathbf{3 a}^{\mathrm{b}}}^{\mathrm{b}}$ \\
\hline 1 & $\mathrm{Cat} .1+\mathrm{PPh}_{3}$ & 98 \\
2 & $\mathrm{Na}_{2} \mathrm{PdCl}_{4}+\mathrm{PPh}_{3}$ & 65 \\
3 & $\mathrm{PdCl}_{2}+\mathrm{PPh}_{3}$ & 81 \\
4 & $\mathrm{Pd}(\mathrm{OAc})_{2}+\mathrm{PPh}_{3}$ & 82 \\
5 & $\mathrm{Na}_{2} \mathrm{PdCl}_{4}+\mathrm{L}+\mathrm{PPh}_{3}$ & $10^{\mathrm{c}}$ \\
6 & $\mathrm{Na}_{2} \mathrm{PdCl}_{4}+\mathrm{L}+\mathrm{dppe}$ & 78 \\
7 & $\mathrm{Na}_{2} \mathrm{PdCl}_{4}+\mathrm{L}+\mathrm{dppf}$ & $15^{\mathrm{c}}$ \\
\hline
\end{tabular}

${ }^{a}$ benzoyl chloride $(1.0 \mathrm{mmol})$, phenyl boronic acid $(0.5 \mathrm{mmol}), \mathrm{K}_{2} \mathrm{CO}_{3}(1.0 \mathrm{mmol})$, complex $\mathbf{1}$ $(0.5 \mathrm{~mol} \%)$, tetraethyl tetra- $t$-butylcalixarene-tetra(oxyacetate) $\mathbf{L}(0.005 \mathrm{mmol}), \mathrm{NaCl}(0.005$ $\mathrm{mmol}), \mathrm{PdCl}_{2}(0.005 \mathrm{mmol}), \mathrm{Pd}(\mathrm{OAc})_{2}(0.005 \mathrm{mmol})$, phosphine ligand $(0.01 \mathrm{mmol})$ and toluene $(2.0 \mathrm{~mL}), 70{ }^{\circ} \mathrm{C}, 24 \mathrm{~h} .{ }^{\mathrm{b}} \mathrm{GC}$ yields by using $\mathbf{3 a}$ as internal standard substances. ${ }^{\mathrm{c}}$ the side product ( 70\% GC yield) was determined by using biphenyl as internal standard substances.

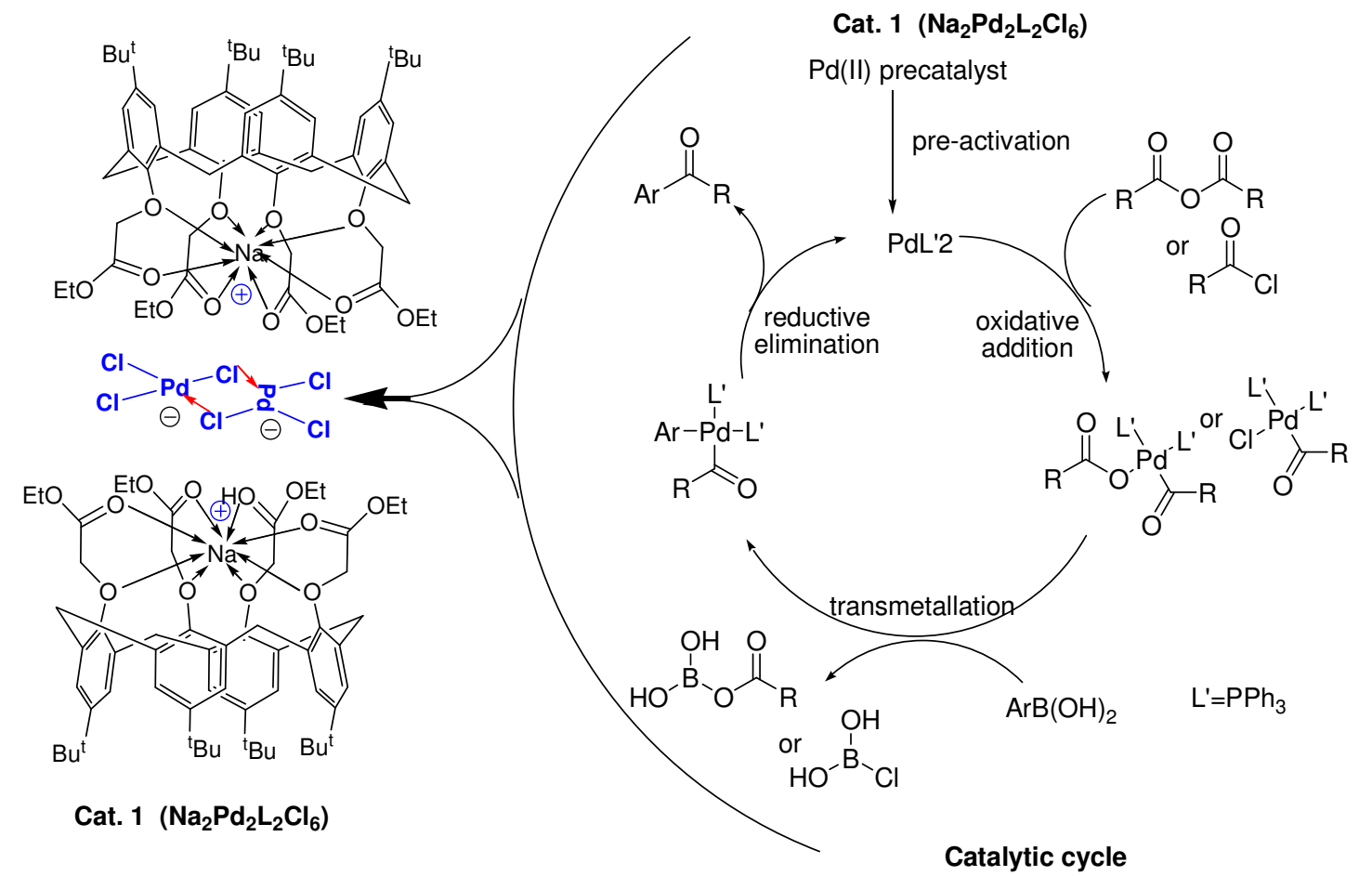

Scheme 3. Possible mechanism. 
Based on the ligature and results obtained above, a possible reaction mechanism was proposed as outlined in Scheme 3. Firstly, Pre-activation of Cat.1 generated the active palladium catalyst $\mathrm{PdL}_{2}$ in the anion of $\mathrm{Pd}_{2} \mathrm{Cl}_{6}{ }^{-}$. We hypothesized that the active palladium catalyst covered on the anion as shown in Scheme 4. The benzoyl chloride $\mathbf{2 a}$ was transported one by one through the cage of tetraethyl tetra-t-butylcalixarene-tetra(oxyacetate) $\mathbf{L}$ to access the active palladium catalyst from two side (Scheme 4). Fast oxidative addition gave the intermediate. After the completion of transmetallation of phenylboronic acid 1a, the catalytic cycle was finished followed by the reductive elimination finally.
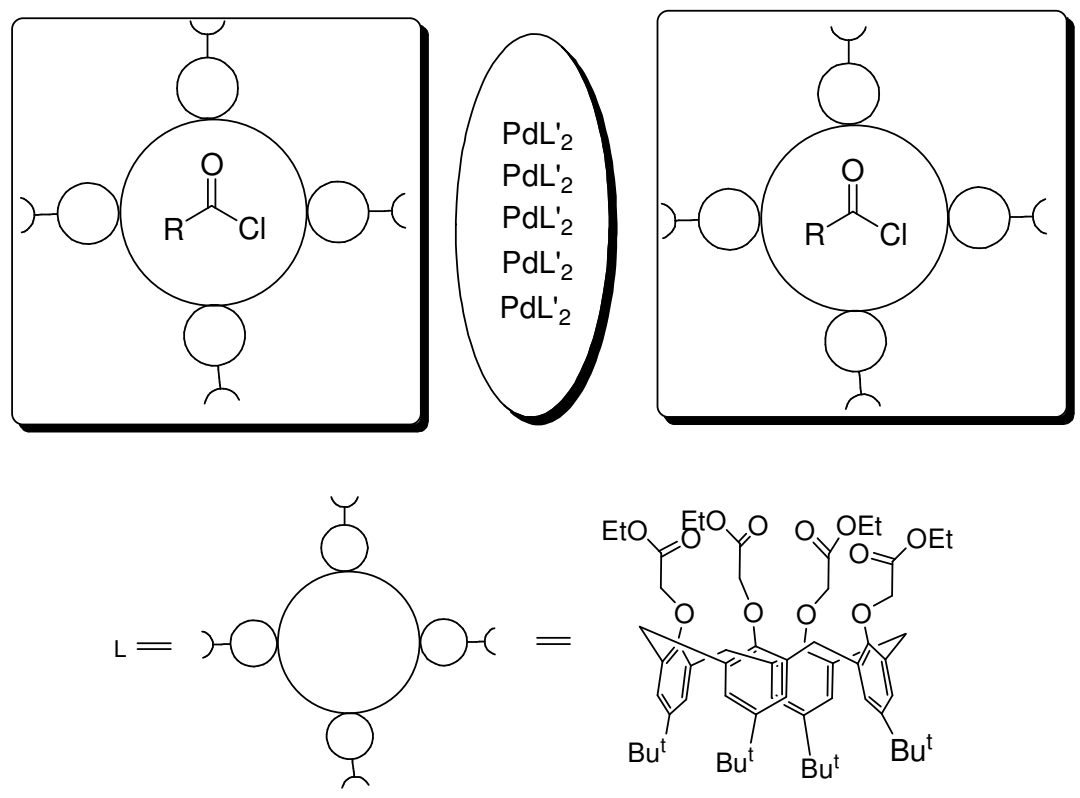

Scheme 4. The role of the tetraethyl $p$-tert-butyl-calix[4]aryl tetraacetate.

It is reliable that the catalytic cycle was finished in the anion part of $\mathrm{Pd}_{2} \mathrm{Cl}_{6}{ }^{-}$since the biphenyl was observed as main product when the ligand $\mathbf{L}$ was mixed with $1 \mathbf{1 a}$ and $\mathbf{2 a}$ (Table 5, entry 5). We resumed that the efficiency of Pd-tetraethyl tetra-t-butylcalixarene-tetra (oxyacetate) complex in the carbonylation reaction could be attributed to its surface properties, and its capacity to bind strongly to benzoyl chloride into its hydrophobic pocket, which enhanced the ability to interact with the reagents and palladium (Scheme 4). ${ }^{18 \mathrm{~d}}$

\section{Conclusions}

In summary, Pd-tetraethyl tetra-t-butylcalixarene-tetra(oxyacetate) complex, in the form of $[\mathrm{NaL}]^{+}\left[\mathrm{PdCl}_{3}\right]^{-}$, catalyzed cross-coupling reactions of various arylboronic acids with benzoyl chloride or acetic anhydride were successfully achieved with low catalyst loading of $0.5 \mathrm{~mol} \%$. This protocol has the advantages of straightforward, easy handling and cheap substrates as 
coupling partner. Most important, the advantage of high functional group tolerance made this method possible to obtain several acetophenones difficult to access by traditional Friedel-Crafts reaction. Moreover, this study provides a particular method to synthesize methyl aryl ketones by acetylation using acylodeboronation reactions.

\section{Experimental Section}

General. Melting points were measured on a XT-5 microscopic apparatus. GC analyses were performed on Agilent 4890D gas chromatograph. ${ }^{1} \mathrm{H}$ NMR and ${ }^{13} \mathrm{C}$ NMR spectra were recorded on a Bruker DPX 400 instrument using $\mathrm{CDCl}_{3}$ or DMSO- $d_{6}$ as the solvent and TMS as the internal standard.

Palladium complex based on a tetraethyl tetra-t-butylcalixarene-tetra(oxyacetate) was synthesized according to the literature. ${ }^{3 \mathrm{~d}}$ All solvents were tried by the standard methods. The chemicals were reagent grade and used without further treatment.

General procedure for the acylodeboronation reaction of arylboronic acids with benzoyl chloride. A $5 \mathrm{~mL}$ flask charged with benzoyl chloride $(1.0 \mathrm{mmol})$, arylboronic acid $(0.5 \mathrm{mmol})$, $\mathrm{K}_{2} \mathrm{CO}_{3}(1.0 \mathrm{mmol})$, complex $1(0.5 \mathrm{~mol} \%, 3.1 \mathrm{mg}), \mathrm{PPh}_{3}(0.01 \mathrm{mmol}, 1.3 \mathrm{mg})$ and toluene $(2.0$ $\mathrm{mL}$ ) was put into a preheated $70{ }^{\circ} \mathrm{C}$ oil bath for an appropriate period of time under air. After the reaction was finished, the reaction mixture was cooled to room temperature, filtered through a short silica column and washed with ethyl acetate. Then the combined filtrates were concentrated in vасио and the residue was purified by flash chromatography (eluent: ethylacetate/petroleum ether). All the products were known compounds and characterized by comparing mp, ${ }^{1} \mathrm{H}$ NMR and ${ }^{13} \mathrm{C}$ NMR spectra with literature.

Benzophenone (3a, Table 2, entry 1). White solid, mp 47-48 ${ }^{\circ} \mathrm{C}$ (lit. $\left.{ }^{21} 47-49^{\circ} \mathrm{C}\right) .{ }^{1} \mathrm{H}$ NMR (400 $\left.\mathrm{Mz}, \mathrm{CDCl}_{3}\right): \delta 7.81-7.78(\mathrm{~m}, 4 \mathrm{H}), 7.59-7.54(\mathrm{~m}, 2 \mathrm{H}), 7.49-7.45(\mathrm{~m}, 4 \mathrm{H}) ;{ }^{13} \mathrm{C}$ NMR $(100 \mathrm{Mz}$, $\left.\mathrm{CDCl}_{3}\right): \delta 196.7,137.4,132.3,129.9,128.1$.

3-Methyl-benzophenone (3b, Table 2, entry 2). Light yellow oil ${ }^{22} .{ }^{1} \mathrm{H}$ NMR (400 Mz, $\left.\mathrm{CDCl}_{3}\right): \delta$ 7.79-7.77 (m, 2H), 7.62 (s, $\left.1 \mathrm{H}\right), 7.57-7.54(\mathrm{~m}, 2 \mathrm{H}), 7.47-7.44(\mathrm{~m}, 2 \mathrm{H}), 7.37-7.34(\mathrm{~m}$, $2 \mathrm{H}), 2.40(\mathrm{~s}, 3 \mathrm{H}) ;{ }^{13} \mathrm{C} \mathrm{NMR}\left(100 \mathrm{Mz}, \mathrm{CDCl}_{3}\right): \delta 196.8,138.0,137.6,137.5,133.4,132.2,130.3$, 130.0, 128.4, 128.1, 127.2, 21.2.

4-Methoxybenzophenone (3c, Table 2 , entry 3 and entry 14). White solid, mp 59-60 ${ }^{\circ} \mathrm{C}$ (lit. ${ }^{23}$ 61-62 $\left.{ }^{\circ} \mathrm{C}\right) .{ }^{1} \mathrm{H}$ NMR $\left(400 \mathrm{Mz}, \mathrm{CDCl}_{3}\right): \delta 7.83-7.80(\mathrm{~m}, 2 \mathrm{H}), 7.76-7.73(\mathrm{~m}, 2 \mathrm{H}), 7.54-7.45(\mathrm{~m}$, $3 \mathrm{H}), 6.96-6.93(\mathrm{~m}, 2 \mathrm{H}), 3.86(\mathrm{~s}, 3 \mathrm{H}) .{ }^{13} \mathrm{C} \mathrm{NMR}\left(100 \mathrm{Mz}, \mathrm{CDCl}_{3}\right): \delta 195.4,163.0,138.1,132.4$, 131.7, 130.0, 129.6, 128.0,113.4, 55.34.

2-bromobenzophenone (3d, Table 2, entry 4). light yellow oil ${ }^{24} .{ }^{1} \mathrm{H}$ NMR (400 Mz, $\left.\mathrm{CDCl}_{3}\right)$ : $\delta$ 7.80-7.78 (m, 2H), 7.62-7.56 (m, 2H), 7.45. ${ }^{13} \mathrm{C}$ NMR $\left(100 \mathrm{Mz}, \mathrm{CDCl}_{3}\right): \delta$ 195.7, 140.5, $136.0,133.7,133.1,131.1,130.1,128.8,128.5,127.1,119.4$. 
2-Chlorobenzophenone (3e, Table 2, entry 5). White solid, mp $42-43{ }^{\circ} \mathrm{C}$ (lit. ${ }^{10 b} 43-45{ }^{\circ} \mathrm{C}$ ). ${ }^{1} \mathrm{H}$ NMR (400 Mz, $\left.\mathrm{CDCl}_{3}\right): \delta$ 7.79-7.77 (m, $\left.2 \mathrm{H}\right), 7.55-7.53(\mathrm{~m}, 1 \mathrm{H}), 7.44-7.38(\mathrm{~m}, 4 \mathrm{H}), 7.31-7.23$ (m, $2 \mathrm{H}) .{ }^{13} \mathrm{C} \mathrm{NMR}\left(100 \mathrm{Mz}, \mathrm{CDCl}_{3}\right): \delta$ 195.4, 138.4, 136.3, 133.6, 131.1, 131.0,130.0, 129.0, 128.5, 126.6 .

3-Chlorobenzophenone (3f, Table 2, entry 6). White solid, mp 81-83 ${ }^{\circ} \mathrm{C}$ (lit. $\left.{ }^{25} .84{ }^{\circ} \mathrm{C}\right) .{ }^{1} \mathrm{H}$ NMR $\left(400 \mathrm{Mz}, \mathrm{CDCl}_{3}\right): \delta$ 7.79-7.77 (m, 3H), 7.66-7.64 (m, 1H), 7.59-7.50 (m, 2H), 7.48-7.46 (m, 2H), 7.43-7.40 (m, 1H). ${ }^{13} \mathrm{C}$ NMR (100 Mz, $\left.\mathrm{CDCl}_{3}\right): \delta 195.0,139.1,136.8,134.4,132.7,132.2$, 130.1, 129.9, 129.7, 129.5, 128.5, 128.3, 128.0.

4-Chlorobenzophenone (3g, Table 2, entry 7). White solid, mp 73-74 ${ }^{\circ} \mathrm{C}$ (lit. $\left.{ }^{26} 73-74{ }^{\circ} \mathrm{C}\right) .{ }^{1} \mathrm{H}$ NMR $\left(400 \mathrm{Mz}, \mathrm{CDCl}_{3}\right): \delta$ 7.77-7.73 $(\mathrm{m}, 4 \mathrm{H}), 7.58-7.56(\mathrm{~m}, 1 \mathrm{H}), 7.51-7.45(\mathrm{~m}, 4 \mathrm{H}) .{ }^{13} \mathrm{C} \mathrm{NMR}$ $\left(100 \mathrm{Mz}, \mathrm{CDCl}_{3}\right): \delta 195.3,138.7,137.0,135.7,132.5,131.1,129.8,128.5,128.2$.

4-Fluoro-benzophenone (3h, Table 2, entry 8). White solid, mp $48-49{ }^{\circ} \mathrm{C}$ (lit. ${ }^{27} 47-49{ }^{\circ} \mathrm{C}$ ). ${ }^{1} \mathrm{H}$ NMR $\left(400 \mathrm{Mz}, \mathrm{CDCl}_{3}\right): \delta$ 7.85-7.75 (m, 4H), 7.58-7.45 (m, 3H), 7.16-7.12 (m, 2H); ${ }^{13} \mathrm{C}$ NMR $\left(100 \mathrm{Mz}, \mathrm{CDCl}_{3}\right): \delta 195.1,166.5,164.0,137.3,133.7,133.6,132.6,132.5,132.3,130.0,128.5$, 128.2, 115.9, 115.2.

4-Acetobenzophenone (3i, Table 2, entry 9). White solid, mp 83-84 ${ }^{\circ} \mathrm{C}$ (lit. ${ }^{28} 83-84{ }^{\circ} \mathrm{C}$ ). ${ }^{1} \mathrm{H}$ NMR (400 Mz, $\left.\mathrm{CDCl}_{3}\right): \delta$ 8.07-8.04 (m, 2H), 7.87-7.85 (m, 2H), 7.82-7.79 (m, 2H), 7.62-7.50 $(\mathrm{m}, 3 \mathrm{H}), 2.67(\mathrm{~s}, 3 \mathrm{H}) .{ }^{13} \mathrm{C} \mathrm{NMR}\left(100 \mathrm{Mz}, \mathrm{CDCl}_{3}\right): \delta 197.5,195.9,141.1,139.4,136.7,133.8$, $130.1,130.0,128.5,128.3,26.8$.

4-Formyl-benzophenone (3j, Table 2, entry 10). White solid, mp 57-59 ${ }^{\circ} \mathrm{C}$ (lit. $\left.{ }^{29} 58-60{ }^{\circ} \mathrm{C}\right) .{ }^{1} \mathrm{H}$ NMR (400 Mz, $\left.\mathrm{CDCl}_{3}\right): \delta 10.1(\mathrm{~s}, 1 \mathrm{H}), 7.95-7.92(\mathrm{~m}, 2 \mathrm{H}), 7.91-7.89(\mathrm{~m}, 2 \mathrm{H}), 7.81-7.79(\mathrm{~m}$, 2H), 7.62-7.58 (m, 1H), 7.52-7.44 (m, 2H); $\left.{ }^{13} \mathrm{C} \mathrm{NMR} \mathrm{(100} \mathrm{Mz,} \mathrm{CDCl}_{3}\right): \delta$ 195.6, 191.6, 142.4, 138.3, 133.0, 130.1, 129.7, 129.2, 128.4.

1-Benzoylnaphthalene (3k, Table 2, entry 11). White solid, mp 74-75 ${ }^{\circ} \mathrm{C}$ (lit. $\left.{ }^{30} 74-76{ }^{\circ} \mathrm{C}\right) .{ }^{1} \mathrm{H}$ NMR (400 Mz, $\left.\mathrm{CDCl}_{3}\right): \delta 8.10-8.08(\mathrm{~m}, 1 \mathrm{H}), 7.94(\mathrm{~d}, J 8.4 \mathrm{~Hz}, 1 \mathrm{H}), 7.93-7.82(\mathrm{~m}, 3 \mathrm{H}), 7.53-$ $7.38(\mathrm{~m}, 7 \mathrm{H}) .{ }^{13} \mathrm{C} \mathrm{NMR}\left(100 \mathrm{Mz}, \mathrm{CDCl}_{3}\right): \delta 197.9,138.2,136.2,133.6,133.2,131.2,130.8$, $130.3,128.7,128.4,127.7,127.2,126.4,125.6,124.3$.

2-Benzoylthiophene (31, Table 2, entry 12). White solid, mp 54-55 ${ }^{\circ} \mathrm{C}$ (lit. ${ }^{31} 56-57{ }^{\circ} \mathrm{C}$ ). ${ }^{1} \mathrm{H}$ NMR $\left(400 \mathrm{Mz}, \mathrm{CDCl}_{3}\right): \delta$ 7.85-7.87 (m, 2H), 7.72-7.71 (m, 1H), 7.64-7.63 (m, 1H), 7.61-7.58 $(\mathrm{m}, 1 \mathrm{H}), 7.50-7.48(\mathrm{~m}, 2 \mathrm{H}), 7.16-7.14(\mathrm{~m}, 1 \mathrm{H}) .{ }^{13} \mathrm{C} \mathrm{NMR}\left(100 \mathrm{Mz}, \mathrm{CDCl}_{3}\right): \delta 188.1,143.5$, $138.0,134.8,134.2,132.2,130.0,129.0,128.3,127.9$.

4-Methyl-benzophenone (3m, Table 2, entry 13). light yellow oil ${ }^{32} .{ }^{1} \mathrm{H}$ NMR (400 Mz, $\left.\mathrm{CDCl}_{3}\right): \delta 8.10-8.08(\mathrm{~d}, J 8.0 \mathrm{~Hz}, 2 \mathrm{H}), 7.79-7.71(\mathrm{~d}, J 7.6 \mathrm{~Hz}, 2 \mathrm{H}), 7.59-7.55(\mathrm{~m}, 1 \mathrm{H}), 7.20(\mathrm{~m}$, $5 \mathrm{H}), 2.43(\mathrm{~s}, 3 \mathrm{H}) ;{ }^{13} \mathrm{C} \mathrm{NMR}\left(100 \mathrm{Mz}, \mathrm{CDCl}_{3}\right): \delta 196.5,165.3,151.0,144.4,143.3,132.2$, $130.3,130.2,130.0,129.5,129.3,129.0,128.2,126.8,125.8,121.8,21.7$.

General procedure for the acylodeboronation reaction of arylboronic acids with acetic anhydride. A $5 \mathrm{~mL}$ flask charged with acetic anhydride $(1.0 \mathrm{mmol})$, arylboronic acid $(0.5$ $\mathrm{mmol}), \mathrm{KHCO}_{3}(1.0 \mathrm{mmol})$, complex 1 (0.5 mol\%, $\left.3.1 \mathrm{mg}\right), \mathrm{PPh}_{3}(0.01 \mathrm{mmol}, 1.3 \mathrm{mg})$ and toluene $(2.0 \mathrm{~mL})$ was evacuated and backfilled with $\mathrm{N}_{2}$ for three times before the reaction was put into a preheated $110{ }^{\circ} \mathrm{C}$ oil bath. After the reaction was finished in $3 \mathrm{~h}$, the reaction mixture 
was cooled to room temperature, filtered through a short silica column and washed with ethyl acetate. Then the combined filtrates were concentrated in vacuo and the residue was purified by flash chromatography (eluent: ethylacetate/petroleum ether). All the products were known compounds and characterized by comparing mp, ${ }^{1} \mathrm{H}$ NMR and ${ }^{13} \mathrm{C}$ NMR spectra with literature.

Acetophenone (5a, Table 4, entry 1). Light yellow oil ${ }^{33}{ }^{1} \mathrm{H}$ NMR $\left(400 \mathrm{Mz}, \mathrm{CDCl}_{3}\right): \delta 7.97$ $7.94(\mathrm{~m}, 2 \mathrm{H}), 7.58-7.54(\mathrm{~m}, 1 \mathrm{H}), 7.48-7.45(\mathrm{~m}, 2 \mathrm{H}), 2.58(\mathrm{~s}, 3 \mathrm{H}) ;{ }^{13} \mathrm{C} \mathrm{NMR}\left(100 \mathrm{Mz}, \mathrm{CDCl}_{3}\right)$ : $\delta 198.5,137.6,133.5,129.0,128.7,26.9$.

3-Methylacetophenone (5b, Table 4, entry 2). Light yellow oil ${ }^{14}{ }^{1} \mathrm{H}$ NMR (400 $\left.\mathrm{Mz}, \mathrm{CDCl}_{3}\right)$ : $\delta$ 7.76-7.73 (m, 2H), 7.35-7.33 (m, 2H), $2.60(\mathrm{~s}, 3 \mathrm{H}), 2.40(\mathrm{~s}, 3 \mathrm{H}) .{ }^{13} \mathrm{C} \mathrm{NMR}\left(100 \mathrm{Mz}, \mathrm{CDCl}_{3}\right)$ : $\delta 198.4,138.3,137.1,133.8,128.7,128.4,125.5,26.6,21.3$.

4-Methoxyacetophenone (5c, Table 4, entry 3). Light yellow oil ${ }^{33}{ }^{1} \mathrm{H} \mathrm{NMR}\left(400 \mathrm{Mz}, \mathrm{CDCl}_{3}\right)$ : $\delta 7.91(\mathrm{~d}, J 8.80 \mathrm{~Hz}, 2 \mathrm{H}), 6.93(\mathrm{~d}, J 8.80 \mathrm{~Hz}, 2 \mathrm{H}), 3.84(\mathrm{~s}, 3 \mathrm{H}), 2.52(\mathrm{~s}, 3 \mathrm{H}) .{ }^{13} \mathrm{C} \mathrm{NMR}(100 \mathrm{Mz}$, $\left.\mathrm{CDCl}_{3}\right): \delta 196.7,163.5,130.5,130.3,113.6,55.4,26.3$.

4-Methylacetophenone (5d, Table 4, entry 4). Light yellow oil ${ }^{33}{ }^{1} \mathrm{H} \mathrm{NMR}\left(400 \mathrm{Mz}, \mathrm{CDCl}_{3}\right)$ : $\delta 7.84(\mathrm{~d}, J 8.04 \mathrm{~Hz}, 2 \mathrm{H}), 7.23(\mathrm{~d}, J 8.04 \mathrm{~Hz}, 2 \mathrm{H}), 2.55(\mathrm{~s}, 3 \mathrm{H}), 2.39$ (s, 3H). ${ }^{13} \mathrm{C} \mathrm{NMR}(100 \mathrm{Mz}$, $\left.\mathrm{CDCl}_{3}\right): \delta$ 197.8, 143.8, 134.7, 129.2, 128.4, 26.5, 21.6.

1-Acetonaphthone (5e, Table 4, entry 5). Light yellow oil ${ }^{33}{ }^{1} \mathrm{H}$ NMR (400 Mz, $\left.\mathrm{CDCl}_{3}\right): \delta 8.74$ $(\mathrm{d}, J 8.60 \mathrm{~Hz}, 1 \mathrm{H}), 7.85(\mathrm{~m}, 3 \mathrm{H}), 7.57(\mathrm{~m}, 1 \mathrm{H}), 7.45(\mathrm{~m}, 2 \mathrm{H}), 2.69(\mathrm{~s}, 3 \mathrm{H}) .{ }^{13} \mathrm{C}$ NMR $(100 \mathrm{Mz}$, $\left.\mathrm{CDCl}_{3}\right): \delta 201.8,135.4,134.0,133.0,130.2,128.7,128.4,128.0,126.4,126.0,124.3,30.0$.

3-Chloroacetophenon (5f, Table 4, entry 6). light yellow oil ${ }^{[34]}{ }^{1} \mathrm{H}$ NMR (400 Mz, $\left.\mathrm{CDCl}_{3}\right): \delta$ 7.93-7.92 (m, 1H), 7.84-7.82 (m, 1H), 7.55-7.53 (m, 1H), 7.43-7.39 (m, 1H), $2.60(\mathrm{~s}$, $3 \mathrm{H}) ;{ }^{13} \mathrm{C} \mathrm{NMR}\left(100 \mathrm{Mz}, \mathrm{CDCl}_{3}\right): \delta$ 196.7, 138.6, 134.9, 133.0, 129.9, 128.4, 126.4, 26.6.

4-Chloroacetophenone (5g, Table 4, entry 7). light yellow oil ${ }^{34}{ }^{1} \mathrm{H}$ NMR (400 Mz, $\left.\mathrm{CDCl}_{3}\right): \delta 7.88(\mathrm{~d}, J 8.8 \mathrm{~Hz}, 2 \mathrm{H}), 7.42(\mathrm{~d}, J 8.8 \mathrm{~Hz}, 2 \mathrm{H}), 2.58(\mathrm{~s}, 3 \mathrm{H}) ;{ }^{13} \mathrm{C} \mathrm{NMR}(100 \mathrm{Mz}$, $\left.\mathrm{CDCl}_{3}\right): \delta 196.8,139.5,135.4,129.7,129.4,128.9,122.9,26.5$.

2-Chloroacetophenone (5h, Table 4, entry 8). Llight yellow oil ${ }^{34}{ }^{1} \mathrm{H}$ NMR (400 $\left.\mathrm{Mz}, \mathrm{CDCl}_{3}\right)$ : $\delta$ 7.56-7.54 (m, 1H), 7.41-7.31 (m, $3 \mathrm{H}), 2.65(\mathrm{~s}, 3 \mathrm{H}) ;{ }^{13} \mathrm{C} \mathrm{NMR}\left(100 \mathrm{Mz}, \mathrm{CDCl}_{3}\right): \delta 200.4$, $139.1,132.0,131.2,130.6,129.4,126.9,30.7$.

1-phenylpropan-1-one (5i, Table 4, entry 9). Light yellow oil ${ }^{33}{ }^{1} \mathrm{H}$ NMR (400 $\left.\mathrm{Mz}, \mathrm{CDCl}_{3}\right)$ : $\delta$ 8.00-7.96 (m, 1H), 7.56-7.44 (m, 2H), 7.09-7.07 (m, $2 \mathrm{H}), 3.0(\mathrm{~m}, 2 \mathrm{H}), 1.22-1.24(\mathrm{~m}, 3 \mathrm{H}) ;{ }^{13} \mathrm{C}$ NMR (100 Mz, $\left.\mathrm{CDCl}_{3}\right): \delta$ 198.5, 137.6, 133.5, 129.0, 128.7, 26.9.

\section{Acknowledgements}

We are grateful to the National Science Foundation of China (Project 21102132, 21172200, 20772114) and the Innovation Fund for Outstanding Scholar of Henan Province (Project 0621001100) for the financial support given to this research. 


\section{References}

1. (a) Nabuurs, S. B.; Wagener, M.; Vlieg, J. J. Med. Chem. 2007, 50, 6507.

http://dx.doi.org/10.1021/jm070593p

PMid:18031000

(b) Tang, G.; Nikolovska-Coleska, Z.; Qiu, S.; Yang, C.; Guo, J.; Wang, S. J. Med. Chem.

$2008,51,717$.

http://dx.doi.org/10.1021/jm701358v

PMid:18237106

2. (a) Li, J.; Jiang, Y.; Tu, P.-F. J. Nat. Prod. 2005, 68, 1802.

http://dx.doi.org/10.1021/np0503471

PMid:16378382

(b) Deng, Y.; Chin, Y. W.; Chai, H.; Keller, W. J.; Kinghorn, A. D. J. Nat. Prod. 2007, 70, 2049.

http://dx.doi.org/10.1021/np070501z

PMid:18076142

(c) Baggett, S.; Mazzola, E. P.; Kennelly, E. J. Stud. Nat. Prod. Chem. 2005, 32, 721.

http://dx.doi.org/10.1016/S1572-5995(05)80067-5

3. (a) Dobashi, Y.; Ohkatsu, Y.; Polym. Degrad. Stab. 2008, 93, 436.

http://dx.doi.org/10.1016/j.polymdegradstab.2007.11.011

(b) Calafat, A. M.; Wong, L. Y.; Ye, X.; Reidy, J. A.; Needham, L. L. Environ. Health

Perspect. 2008, 116, 893.

http://dx.doi.org/10.1289/ehp.11269

PMid:18629311 PMCid:245315

(c) Nohynek, G. J.; Schaefer, H. Regul. Toxicol. Pharmacol. 2001, 33, 285.

http://dx.doi.org/10.1006/rtph.2001.1476

PMid:11407932

4. (a) Franck, H. G.; Stadelhofer, J. W. Industrial Aromatic Chemistry; Springer: Berlin, 1988. http://dx.doi.org/10.1007/978-3-642-73432-8

(b) Roberts, S. M.; Poignant, G. Catalysts for Fine Chemical Synthesis, Vol.1: Hydrolysis, Oxidation and Reduction; Wiley-VCH: Weinheim, 2002.

(c) Surburg, H.; Panten, J. Common Fragrance and Flavor Materials, 5th Ed.; Wiley-VCH: Weinheim, Germany, 2006.

http://dx.doi.org/10.1002/3527608214

(d) Romines, K. R.; Freeman, G. A.; Schaller, L.T.; Cowan, J. R.; Gonzales, S. S.; Tidwell J.

H.; Andrews, C. W.; Stammers, D. K.; Hazen, R. J.; Ferris, R. G.; Short, S. A.; Chan, J. H.;

Boone, L. R. J. Med. Chem. 2006, 49, 727.

http://dx.doi.org/10.1021/jm0506701

PMid:16420058

(e) Masson, P. J.; Coup, D.; Millet, J.; Brown, N. L. J. Biol. Chem. 1994, 270, 2662. 
(f) De Kimpe, N.; Keppens, M.; Fonck, G. Chem. Commun. 1996, 635.

http://dx.doi.org/10.1039/cc9960000635

5. (a) Negishi, E.; Yosida, T. J. Org. Chem. 1975, 40, 1676.

(b) Cho, C. S.; Uemara, S. J. Organomet. Chem. 1993, 443, 253.

(c) Haddach, M.; McCarthy, J. R. Tetrahedron Lett. 1999, 40, 3109.

(d) Bumagin, N. A. Tetrahedron. Lett. 1999, 40, 3057.

http://dx.doi.org/10.1016/S0040-4039(99)00364-0

(e) Chen, H. Org. Lett. 2000, 2, 1649.

http://dx.doi.org/10.1021/o1000013h

PMid:10880192

(f) Gooben, L. J.; Ghosh, K. Angew. Chem. Int. Ed. 2001, 40, 3458.

http://dx.doi.org/10.1002/1521-3773(20010917)40:18<3458::AID-ANIE3458>3.0.CO;2-0

(g) Yamamoto, A. Bull. Chem. Soc. Jpn. 2001, 74, 371.

http://dx.doi.org/10.1246/bcsj.74.371

(h) Wang, J.; Zhang, Y.; Fu, Y. Bull. Chem. Soc. Jpn 2002, 75, 1381.

http://dx.doi.org/10.1246/bcsj.75.1381

(i) Urawa, Y.; Ogura, K. Tetrahedron Lett. 2003, 44, 271.

http://dx.doi.org/10.1016/S0040-4039(02)02501-7

(j) Eddarir, S.; Cotelle, N.; Rolando C. Tetrahedron. Lett. 2003, 44, 5359.

http://dx.doi.org/10.1016/S0040-4039(03)01140-7

6. (a) Tatamidani, H.; Chatani, N. J. Org. Chem. 2004, 69, 5615.

http://dx.doi.org/10.1021/jo0492719

PMid:15307730

(b) Tatamidani, H.; Kakiuchi, F.; Chatani, N. Org. Lett. 2004, 6, 3597.

http://dx.doi.org/10.1021/o10485130

PMid:15387557

(c) Liebeskind L. S. J. Org. Chem. 2004, 69, 3554.

http://dx.doi.org/10.1021/jo049964p

PMid: 15132570

(d) Xin, B.; Zhang, Y.; Cheng, K. J. Org. Chem. 2006, 71, 5725.

http://dx.doi.org/10.1021/jo060749d

PMid:16839154

(e) Nishihara, A.; Inoue, Y.; Fujisawa, M.; Takagi, K. Synlett 2005, 2309.

http://dx.doi.org/10.1055/s-2005-872661

(f) Gooben, L. J.; Koley, D.; Thiel, W. J. Am. Chem. Soc. 2005, 127, 11102.

http://dx.doi.org/10.1021/ja052435y

PMid:16076218

(g) Bandgar, B. P.; Patil, A. V. Tetrahedron Lett. 2005, 46, 7627.

http://dx.doi.org/10.1016/j.tetlet.2005.08.111 
7. (a) Polackova, V.; Toma, S.; Augustinova I. Tetrahedron 2006, 62, 11675.

http://dx.doi.org/10.1016/j.tet.2006.09.055

(b) Liu, G.; Cai, M. Catal. Commun. 2007, 8, 251.

http://dx.doi.org/10.1016/j.catcom.2006.06.015

(c) Xin, B.; Cheng, K. Synthesis 2007, 1970.

(d) Kekeli, E. K.; Xu, H.; Wolf, C. Tetrahedron Lett. 2008, 49, 5773.

http://dx.doi.org/10.1016/j.tetlet.2008.07.115

(e) Maddali, L. N. R.; Varadhachari, V.; Priyabrata, D. Tetrahedron Lett. 2010, 51, 4975.

http://dx.doi.org/10.1016/j.tetlet.2010.07.074

(f) Zhang, L.; Wu, J. L.; Shi, L. J.; Xia, C. G.; Li, F. W. Tetrahedron Lett. 2011, 52, 3897.

http://dx.doi.org/10.1016/j.tetlet.2011.05.079

8. (a) Olah, G. A.; Friedel-Crafts Chemistry; Wiley: New York, 1973.

(b) Song, C. E.; Shim, W. H.; Roh, E. J.; Choi, J. H. Chem. Commun. 2000, 1695.

http://dx.doi.org/10.1039/b005335j

(c) Furstner, A.; Voigtlander, D.; Schrader, W.; Giebel, D.; Reetz, M. T. Org. Lett. 2001, 3, 417.

http://dx.doi.org/10.1021/o10069251

PMid:11428028

(d) Ross, J.; Xiao J. Green Chem. 2002, 4, 129.

http://dx.doi.org/10.1039/b109847k

(e) Gmouh, S.; Yang, H.; Vaultier, M. Org. Lett. 2003, 5, 2219.

http://dx.doi.org/10.1021/o1034529n

PMid:12816413

(f) Fillion, E.; Fishlock, D.; Wilsily, A.; Goll, J.M. J. Org. Chem. 2005, 70, 1316.

http://dx.doi.org/10.1021/jo0483724

PMid:15704966

9. (a) Cai, M.; Zheng, G.; Zha, L.; Peng, J. Eur. J. Org. Chem. 2009, 10, 1585.

http://dx.doi.org/10.1002/ejoc.200801253

(b) Khedkar, M. V.; Tambade, P.J.; Qureshi, Z. S.; Bhanage, B. M. Eur. J. Org. Chem. 2010, 36, 6981 .

http://dx.doi.org/10.1002/ejoc.201001134

(c) O'Keefe, B. M.; Simmons, N.; Martin, S. F. Org. Lett. 2008, 10, 5301.

(d) Ahmed, M. S. M.; Mori, A. Org. Lett. 2003, 5, 3057.

http://dx.doi.org/10.1021/o1035007a

PMid:12916980

(e) Liang, B.; Huang, M.; You, Z.; Xiong, Z.; Lu, K.; Fathi, R.; Chen, J.; Yang, Z. J. Org.

Chem. 2005, 70, 6097.

http://dx.doi.org/10.1021/jo050498t

PMid:16018709 
(f) Peng, C.; Cheng, J.; Wang, J. J. Am. Chem. Soc. 2007, 129, 8708.

http://dx.doi.org/10.1021/ja073010+

PMid:17589999

(g) Lee, S. W.; Lee, K.; Seomoon, D.; Kim, S.; Kim, H.; Shim, E.; Lee, M. J. Org. Chem.

2004, 69, 4852.

http://dx.doi.org/10.1021/jo0495790

PMid:15230616

10. (a) Bensari, A.; Zaveri, N. T. Synthesis 2003, 267.

(b) Chan, C. W.; Zhou, Z.; Chan, A. S. C.; Yu, W. Y. Org. Lett. 2010, 12, 3926.

http://dx.doi.org/10.1021/ol101618u

PMid:20704334

(c) Jia, X.; Zhang, S.; Wang, W.; Luo, F.; Cheng, J.; Org. Lett. 2009, 11, 3120.

http://dx.doi.org/10.1021/o1900934g

PMid:19552400

(d) Kochi, T.; Urano, S.; Seki, H.; Mizushima, E.; Sato, M.; Kakiuchi, F. J. Am. Chem. Soc. 2009, 131, 2792.

http://dx.doi.org/10.1021/ja8097492

PMid:19209853

(e) Xiao, F.; Shuai, Q.; Zhao, F.; Basle, O.; Deng, G.; Li, C. J. Org. Lett. 2011, 13,1614.

http://dx.doi.org/10.1021/ol200017a

PMid:21355624

(f) Zhang, L.; Ang, G. Y.; Chiba, S. Org. Lett. 2011, 13, 1622.

http://dx.doi.org/10.1021/ol200425c

PMid:21381689

g) Yang, Y.; Chen, L.; Zhang, Z.; Zhang, Y. Org. Lett. 2011, 13, 1342. (

http://dx.doi.org/10.1021/ol200025k

PMid:21332150

h) Wu, X. F.; Anbarasan, P.; Neumann, H.; Beller, M. Angew. Chem. Int. Ed. 2010, 49, 7316. http://dx.doi.org/10.1002/anie.201003895

PMid:20740517

11. (a) Wu, S.; Ma, H.; Lei, Z. Tetrahedron 2010, 66, 8641.

http://dx.doi.org/10.1016/j.tet.2010.09.035

(b) Xing, D.; Guan, B.; Cai, G.; Fang, Z.; Yang, L.; Shi, Z. Org. Lett. 2006, 8, 693.

http://dx.doi.org/10.1021/ol052830t

PMid:16468744

(c) Guan, B.; Xing, D.; Cai, G.; Wan, X.; Yu, N.; Fang, Z.; Yang, L.; Shi, Z. J. Am. Chem.

Soc. 2005, 127, 18004.

http://dx.doi.org/10.1021/ja055398j

PMid:16366542 
12. (a) Wong, Y. C.; Parthasarathy, K.; Cheng, C. H. Org. Lett. 2010, 12, 1736.

http://dx.doi.org/10.1021/ol1003252

PMid:20334426

(b) Zhao, B. W.; Lu, X. Y. Tetrahedron Lett. 2006, 47, 6765.

http://dx.doi.org/10.1016/j.tetlet.2006.07.074

(c) Yu, A.; Li, J.; Cui, M.; Wu, Y. Synlett 2007, 3063.

(d) Lindh, J.; Sjoberg, P. J. R.; Larhed, M. Angew. Chem. In. Ed. 2010, 49, 7733.

(e) Takemiya, A.; Hartwig, J. F. J. Am. Chem. Soc. 2006, 128, 4800.

13. (a) Álvarez-Bercedo, P.; Flores-Gaspar, A.; Correa, A.; Martin, R. J. Am. Chem. Soc. 2010, $132,466$.

http://dx.doi.org/10.1021/ja909811t

PMid:20038097

(b) Ruan J.; Saidi O.; Iggo J.A.; Xiao J. J. Am. Chem. Soc. 2008, 130,10510.

http://dx.doi.org/10.1021/ja804351z

PMid:18636731

14. (a) Liu, S.; Berry, N.; Thomson, N.; Pettman, A.; Hyder, Z.; Mo, J.; Xiao, J. J. Org. Chem. 2006, 71, 7467.

http://dx.doi.org/10.1021/jo0609632

PMid:16958544

(b) Mo, J.; Xiao, J.; Angew. Chem. Int. Ed. 2006, 45, 4152.

http://dx.doi.org/10.1002/anie.200600799

PMid:16718713

(c) Mo, J.; Xu, L.; Xiao, J. J. Am. Chem. Soc. 2005, 127, 751.

http://dx.doi.org/10.1021/ja0450861

PMid:15643901

(d) Ruan, J.; Li, X.; Saidi, O.; Xiao, J. J. Am. Chem. Soc. 2008, 130, 2424.

http://dx.doi.org/10.1021/ja0782955

PMid:18232688

(e) Hansen, A. L.; Skydstrup, T. J. Org. Chem. 2005, 70, 5997.

http://dx.doi.org/10.1021/jo050669u

PMid:16018696

(f) Tivola, P. B.; Deagostino, A.; Prandi, C.; Venturello, P. Org. Lett. 2002, 4,1275.

http://dx.doi.org/10.1021/ol0255817

(g) Tsubouchi, A.; Onishi, K.; Takeda, T. J. Am. Chem. Soc. 2006, 128,14268.

http://dx.doi.org/10.1021/ja0658822

PMid:17076495

15. Al-Masum, M.; Ng, E.; Wai, M. C. Tetrahedron Lett. 2011, 52, 1008.

http://dx.doi.org/10.1016/j.tetlet.2010.12.085 
16. Homden, D. M.; Redshaw, C. Chem. Rev. 2008, 108, 5086.

http://dx.doi.org/10.1021/cr8002196

PMid:18956902

17. (a) Itagaki, M.; Suzukamo, G.; Nomura, K. Bull. Chem. Soc. Jpn. 1998, 71, 79.

http://dx.doi.org/10.1246/bcsj.71.79

(b) Monnereau, L.; Semeril, D.; Matt, D.; Toupet, L.; Motac, A. J. Adv. Synth. Catal. 2009, $351,1383$.

http://dx.doi.org/10.1002/adsc.200800759

18. (a) Ohto, K.; Ishibashi, H.; Kawakita, H.; Inoue, K.; Oshima T. J. Incl. Phenom. Macrocycl. Chem. 2009, 65, 111. http://dx.doi.org/10.1007/s10847-009-9639-1

(b) Chang, S. K.; Cho, I. J. Chem. Soc., Perkin Trans. 1986, 211.

(c) McKervey, M. A.; Seward, E. M.; Ferguson, G.; Ruhl, B.; Harris, S. J. Chem. Soc., Chem. Commun. 1985, 388.

(d) Mu, B.; Li, J. Y.; Han, Z. X.; Wu, Y. J. J. Organomet. Chem. 2012, 700, 117.

http://dx.doi.org/10.1016/j.jorganchem.2011.11.024

19. Zhou, Z. X.; Xing, Y. J.; Lu, K.; Wu, Y. J. Chin. Chem. Lett. 1998, 9, 1067.

20. (a) Kakino, R.; Narahashi, H.; Shimizu, I.; Yamamoto, A. Bull. Chem. Soc. Jpn 2002, 75, 1333.

http://dx.doi.org/10.1246/bcsj.75.1333

(b) Goossen, L. J.; Ghosh, K. Eur. J. Org. Chem. 2002, 19, 3254.

http://dx.doi.org/10.1002/1099-0690(200210)2002:19<3254::AID-EJOC3254>3.0.CO;2-6

21. Blicke, F. F.; Schulmann, E. L. J. Am. Chem. Soc. 1954, 76, 1226.

http://dx.doi.org/10.1021/ja01634a006

22. Brown, H. C.; Young, H. L. J. Org. Chem. 1957, 22, 719.

http://dx.doi.org/10.1021/jo01358a001

23. Kulp, S. S.; McGee, M. J. J. Org. Chem.1983, 48, 4097. http://dx.doi.org/10.1021/jo00170a044

24. Lerebours, R.; Camacho-Soto, A.; Wolf, C. J. Org. Chem. 2005, 70, 8601. http://dx.doi.org/10.1021/jo051257o

PMid:16209619

25. Clark, M. T.; Hendley, E. C.; Neville, O. K. J. Am. Chem. Soc. 1955, 77, 3280. http://dx.doi.org/10.1021/ja01617a038

26. Greene, F. D.; Gilman, R. V.; James, C. C.; Richard, D. G. J. Org. Chem. 1960, 25, 1790. http://dx.doi.org/10.1021/jo01080a031

27. Morley, J. O. Synthesis 1977, 54. http://dx.doi.org/10.1055/s-1977-24276

28. Zelinski, R. P.; Turnquest, B. W.; Martin, E. C. J. Am. Chem. Soc. 1951, 73, 5521. http://dx.doi.org/10.1021/ja01156a001 
29. Suhana, H.; Srinivasan, P.C. Synth. Commun. 2003, 33, 3097. http://dx.doi.org/10.1081/SCC-120023419

30. Henry, R. H.; Nunn, L. G. J. J. Org. Chem.1947, 12, 540. http://dx.doi.org/10.1021/jo01168a008

31. Rao, M. L. N.; Venkattesh, V.; Banerjee, D. Tetrahedron 2007, 63, 12917. http://dx.doi.org/10.1016/j.tet.2007.10.047

32. Wu, S.; Ma, H.; Lei, Z. Tetrahedron 2010, 66, 8641. http://dx.doi.org/10.1016/j.tet.2010.09.035

33. Ruan, J.; Iggo, J. A.; Berry, N. G.; Xiao, J. J. Am. Chem. Soc. 2010, 132, 16689. http://dx.doi.org/10.1021/ja1081926 PMid:21028842

34. Horton, W. J.; Robertson, D. E. J. Org. Chem. 1960, 25, 1016. http://dx.doi.org/10.1021/jo01076a039 